\title{
A Frictional Cooling Demonstration Experiment with Protons
}

\author{
R. Galea $\dagger$, A. Caldwell $\dagger \ddagger$, L. Newburgh $\uparrow$ \\ $\dagger$ Nevis Laboratories, Columbia University, Irvington, NY, USA. \\ $\ddagger$ Max-Planck-Institut für Physik, München, Deutschland. \\ ฯ Barnard College, Columbia University, New York, NY, USA.
}

\begin{abstract}
Muon cooling is the main technological obstacle in the building of a muon collider. A muon cooling scheme based on Frictional Cooling holds promise in overcoming this obstacle. An experiment designed to demonstrate the Frictional Cooling concept using protons was undertaken. Although the results were inconclusive in the observation of cooling, the data allowed for the qualification of detailed simulations which are used to simulate the performance of a muon collider.
\end{abstract}

\section{Introduction}

The basic idea of Frictional Cooling 1 is to bring the charged particles into a kinetic energy range where the energy loss per unit distance increases with kinetic energy. In its application to a possible muon collider, the cooling channel is placed in a solenoidal field to contain the muons. A constant accelerating force can be applied to the muons resulting in an equilibrium energy. A sample $\mathrm{dT} / \mathrm{dx}$ curve, where $\mathrm{T}$ is the kinetic energy, is shown in Fig. 1 The desired condition can be met for kinetic energies below a few $\mathrm{KeV}$ or kinetic energies beyond about $200 \mathrm{MeV}$. At the high energy end, the change in $\mathrm{dT} / \mathrm{dx}$ with energy is only logarithmic, whereas it is approximately proportional to the speed at low energies. Below the $\mathrm{dT} / \mathrm{dx}$ peak, muons are too slow to ionize the atoms in the stopping medium. The processes leading to energy loss; excitation, elastic scattering on nuclei and charge exchange reactions, yield differences for $\mu^{+}$and $\mu^{-}$. Operating in this energy regime, an electric field can be applied which compensates for the energy loss. Several issues become apparent:

- $\mathrm{dT} / \mathrm{dx}$ is very large in this region of kinetic energy, so we need to work with a low average density (i.e., gas) in order to have a reasonable electric field strength.

- Muonium formation $\left(\mu^{+}+\right.$Atom $\rightarrow \mu e+$ Atom $\left.^{+}\right)$is significant at low $\mu^{+}$energies. In fact, the muonium formation cross section, in this en- 
ergy range, dominates over the electron stripping cross section in all gases except helium [2].

- There is a measured difference in the $\mu^{+}$and $\mu^{-}$energy loss rates near the peak of the $\mathrm{dT} / \mathrm{dx}$ spectrum (Barkas effect 3 ). This effect is assumed to be due to extra processes for $\mu^{+}$, such as charge exchange.

- A possibly fatal problem for $\mu^{-}$is the efficiency loss resulting from muon capture $\left(\mu^{-}+\right.$Atom $\rightarrow \mu$ Atom $\left.+e^{-}\right)$. The cross section for this process has been calculated up to $\mathrm{T}=80 \mathrm{eV}[4$ but has not been measured. The measurement of this cross section is a critical path item to the realization of a muon collider based on Frictional Cooling. Hydrogen and helium are the best candidate media.

Despite some reported evidence for Frictional Cooling [1, it is clear that much more information is needed if this is to become a useful technique for phase space reduction of beams. We have therefore planned a series of experiments to understand the Frictional Cooling process in detail. The results described in this paper are the first measurements in this program.

As a first step, we plan to study the emittance achievable for protons in such a scheme. The behavior exhibited in Fig. 1 is typical for all charged particles. The Frictional Cooling approach should work for a variety of charged particles, including protons (the stopping power for protons in helium is shown in Fig. (2).

An experiment was performed using protons in order to demonstrate the behavior of charged particles in this low energy regime. This experiment had several goals: demonstrating frictional cooling; benchmarking the simulations, and employing many of the experimental components, detectors, etc., which would be needed in future experimentation with muons.

Using protons simplifies the experiment considerably, as they are easily produced and are stable particles. A time of flight experiment was devised employing start and stop detectors, an electric field and a gas cell with thin entrance and exit windows.

\section{RAdiological Research Accelerator Facility (RARAF)}

RARAF is dedicated to research in radio-biology and radiological physics and is located at Nevis Laboratories, in Irvington, N.Y. ${ }^{1}$ RARAF has a $4 \mathrm{MeV}$ Van de Graaff accelerator, which produces beams of positive light ions. The beam provided by RARAF for this experiment was diatomic hydrogen with one electron stripped $\left(H_{2}^{+}\right)$in the energy range of $1.3-1.6 \mathrm{MeV}$. The $H_{2}^{+}$breaks up in the first trigger detector resulting in an effective initial beam of protons with energies in the range of $650-800 \mathrm{KeV} . \mathrm{H}_{2}^{+}$was used since the ion source and accelerator tube have higher efficiency for $\mathrm{H}_{2}^{+}$than for protons at the lower energy reach of the accelerator. The demands made on the beam were not

\footnotetext{
${ }^{1}$ http://www.raraf.org
} 
strict. In fact the beam was not focused in order to reduce currents to a level $(1-15 \mathrm{pA})$ suitable for our first trigger detector.

\section{Detailed Simulations}

The tracking of protons through various materials was implemented using standalone fortran code. Muonium formation, $\mu^{-}$capture, muon decay and processes specifically affecting muon energy loss are included in this simulation. Although the detailed simulations were written with muons in mind, the program can track any charged particle.

The two processes which concern the proton simulations are the electronic and nuclear energy loss processes. Hydrogen formation was not implemented.

The energy loss from nuclear scattering of protons with kinetic energies below $50 \mathrm{KeV}$ was calculated and coded using the Everhart et al. [5] prescription for generating scattering. Individual nuclear scatters are simulated. For energies above $50 \mathrm{KeV}$, the Born Approximation is used. This procedure was compared with the $\mathrm{dT} / \mathrm{dx}$ tables from $\mathrm{NIST}^{2}$ and showed excellent agreement. The electronic energy loss is treated as continuous and the data are taken from the NIST tables.

The breakup of the $H_{2}^{+}$was not simulated. The SRIM $[6]$ program was used to simulate the energy loss of the protons through the $9 \mu \mathrm{m}$ of silicon in the timing detector. The transmitted proton energy spectrum from SRIM was then used as an input to the detailed simulations of the gas cell.

Only a fraction of the delivered protons achieved the equilibrium energy in the amount of gas available. The rest were too energetic or were lost due to acceptance issues.

\section{Time of Flight (TOF) Experiment Setup}

Figure 3 shows the experimental setup. The proton beam was first collimated through a $1 \mathrm{~mm}$ hole separating the RARAF beam line from the experimental section. This collimator, while reducing the delivered current to the experiment, also acted as a baffle protecting the beam line vacuum from degradation as a result of helium gas which leaked from the gas cell.

The proton beam then hit the first timing detector which produced a stop signal for the TOF measurement. The first detector consisted of a D Series planar totally depleted silicon surface barrier detector from ORTEC ${ }^{3}$. Frictional Cooling operates in the region below the ionization peak. Hence from Fig. 2 one can see that only the protons below $\mathcal{O}(100) \mathrm{KeV}$ had the possibility to be cooled. The silicon detector acted to degrade the beam energy, adding energy spread, while also providing the trigger.

\footnotetext{
${ }^{2}$ http://www.nist.gov

${ }^{3}$ ORTEC: http://www.ortec-online.com
} 
After drifting $4.4 \mathrm{~cm}$ the protons entered the gas cell through a thin window. The windows consisted of $\mathrm{a} \sim 20 \mathrm{~nm}$ thick carbon film on a nickel grid with a $55 \%$ open area ratio and a diameter of $2.3 \mathrm{~mm}$, as specified by $\mathrm{SPI}^{4}$. The windows were inspected and it was discovered that in a few grid spacings the carbon was perforated. The windows were epoxied between two precision washers and the sandwich arrangement was further epoxied to the window holders, designed and built at Nevis Laboratories (See Fig. 4).

The gas cell itself and the support structure for the accelerating grid which surrounded the gas cell were made of Teflon (See Fig. [5). The supporting structure needed to be non-conducting and Teflon was selected as it has a low vapor pressure.

The gas flow was controlled through a Piezoelectric valve. A calibration was performed ahead of time, to determine the gas flow rate as a function of applied voltage, but was found to be unstable in situ. During data taking the Piezoelectric valve had to be continuously adjusted manually to maintain a constant cell pressure. There were three feedthroughs into the gas cell: one was used for the pressure measurement, one for the gas intake and one to open the gas cell to the vacuum while evacuation was taking place. The system was in continuous flow as a result of the small imperfections in the windows and the natural permeability of helium. A Pirani gauge was used to measure the pressure in the gas cell. This type of gauge is sensitive to the nature of the gas. Unfortunately its sensitivity for helium pressure measurements is limited in the desired range of operating conditions. As a result, the operating pressure was estimated for values greater than $0.004 \mathrm{~atm}$.

The accelerating grid consisted of 30 copper rings with thin $(1 \mathrm{~mm})$ Teflon separators. The rings were connected in series by a resistor chain resulting in a uniform accelerating field of $60 \mathrm{KV} / \mathrm{m}$. The distance between the windows in the gas cell was $9.2 \mathrm{~cm}$. The gas cell was shorter than the accelerating grid by $7.3 \mathrm{~cm}$, which provided for a short reacceleration field for protons exiting the gas cell. Those protons which were not sufficiently degraded by the silicon and were not in the energy range suitable for stopping were minimally affected by the small reacceleration field.

The second timing detector, in Fig. B was a Micro Channel Plate (MCP) detector $^{5}$. There were two plates with a total potential difference across them of $1600 \mathrm{~V}$.

\section{Trigger and Data Acquisition}

The trigger consisted of a coincidence between the two timing detectors. The Data Acquisition (DAQ) chain was based on fast NIM logic modules and a CAMAC TDC (See Fig. 6). The TDC had a maximum range of $800 \mathrm{~ns}$. The silicon detector singles rate was nominally $40 \mathrm{KHz}$ and was kept below $100 \mathrm{KHz}$ in order to reduce the effect of pile-up and to avoid potential damage to the

\footnotetext{
${ }^{4}$ Structure Probe Inc.: http://www.2spi.com

${ }^{5}$ North Night Vision Technology Co., Ltd. Nanjing, China.
} 
detector. The CAMAC readout system could be readout at a maximum rate of $1 \mathrm{KHz}$. Hence the signal from the silicon detector was discriminated and then digitally delayed by $\sim 700 \mathrm{~ns}$, an amount comparable to the range of the TDC, and used as the stop signal. The rate on the MCP detector was much lower since its geometric acceptance is very small. The MCP signal was used to trigger the common start of the DAQ system, thereby removing potential dead time due to the DAQ readout. It was noticed that noise pulses from the MCP were associated with multiple pulses. A veto was introduced to reduce this noise by requiring that the MCP triggered pulse be isolated in a window of $100 \mathrm{~ns}($ see Fig. 6). In other words, the event was rejected if more than one MCP pulse occurred within 100 ns. The trigger is illustrated in Fig. [

\section{Datasets}

Table 1 summarizes the datasets taken. $H_{2}^{+}$with $\mathrm{T}=1.44 \mathrm{MeV}$ was the nominal running condition. A higher energy, $\mathrm{T}=1.6 \mathrm{MeV}$, run was taken for comparison. At the higher energy, no cooled protons were expected. Other lower statistic runs were taken at $\mathrm{T}=1.3 \mathrm{MeV}$ and $\mathrm{T}=1.5 \mathrm{MeV}$. Runs were taken with the accelerating grid on and off and the gas flow was turned on and off for calibration and monitoring purposes.

\section{Calibrations}

The TDC was calibrated using a pulse generator and gate delay generator as input to the DAQ chain. The absolute calibration for the time offset, taking into account the time delays introduced by the detectors, electronics and cables, was found from three high statistics data runs corresponding to three different distances separating the two timing detectors. The nominal $\mathrm{H}_{2}^{+}$beam energy of $1.44 \mathrm{MeV}$ was used for these three calibration runs. For these data sets the entire gas cell and accelerating grid structures were removed from the experimental section.

As a result of the trigger configuration illustrated in Fig. [7 increasing distance of flight led to a smaller TDC value (see Fig. 8). Figure 8 also indicates a long tail of background which is fit by an exponential with a large time constant. The tails of the distributions were subtracted before proceeding with the analysis. The peaks were then fit with a gaussian and the means were plotted against the separation distance in order to extract the offset for the DAQ system. The calibration is shown in Fig. 9]

The data is compared to the Monte Carlo (MC) expectation in Fig. 10 The data distribution is broader than the MC expectation but there is good agreement on the location of the peak. The peaks of the distributions correspond approximately to the flight time required for a proton with the most probable kinetic energy. The measured time distribution can be better fitted by a convolution of the true time distribution with a gaussian, whose $\sigma$ represents the 
timing resolution of the system.

$$
P\left(t_{\text {measured }}\right)=\int P_{M C}\left(t_{\text {true }}\right) \cdot \frac{1}{\sigma \sqrt{2 \pi}} \exp \left(\frac{-\left(t_{\text {measured }}-t_{\text {true }}\right)^{2}}{2 \sigma^{2}}\right) \mathrm{d} t_{\text {true }}
$$

Fits of the three time distributions determined that $\sigma \approx 17 \mathrm{~ns}$.

\section{Analysis}

The nominal $H_{2}^{+}$beam energy for the analysis was $1.44 \mathrm{MeV}$. Since the breakup of the $\mathrm{H}_{2}^{+}$is not simulated and there were uncertainties in the beam energy calibration, the first step was to determine the kinetic energy of the incoming protons. This was possible using the calibration runs which were taken without the gas cell and accelerating grid structures. From the calibration plot in Fig. 9 one can extract not only the T0 offset needed to reconstruct the TDC measurements but also the slope of the distribution. The slope of the distribution is representative of a velocity. The slope of $0.502 \mathrm{~cm} / \mathrm{ns}$ represents the velocity for a proton with the most probable kinetic energy after the silicon detector $(136 \mathrm{KeV})$. The incoming energy was varied in the simulations such that the output of the SRIM calculations agreed with the values observed in the data. As shown in Fig. 11] the transmitted energy is a strong function of the incoming energy. An initial proton energy of $721 \mathrm{KeV}$ is needed to produce a most probable transmitted energy of $136 \mathrm{KeV}$.

The next step was to determine the effect of the gas cell windows. With the gas cell and accelerating grid in the beam line, a data run was taken without flowing helium gas in the cell and leaving the grid off. In this way the only difference in the TOF distribution resulted from the extra energy loss of the protons in the entrance and exit windows of the gas cell. This data is plotted in Fig. 12 and compared to the simulation of various window thicknesses. An effective carbon window thickness of $350 \mathrm{~nm}$ reproduced the data much better than the quoted thickness of $20 \mathrm{~nm}$. The apparent thickness of $350 \mathrm{~nm}$ was

more than an order of magnitude larger than what was expected. The effect of this thick window was to change the expected TOF distributions by adding an effective lower energy threshold for protons to get through the system. Hence, no protons which would result in a TOF greater than $400 \mathrm{~ns}$ were expected to penetrate the exit window. This greatly reduced the possibility of observing cooled protons.

Finally, the pressure of the gas had to be determined from the data since the pressure gauge was not precise. After adding the gas the $\mathrm{MC}$ was tuned to extract the pressure of the helium gas inside the gas cell. This is shown in Fig. 13. The MC was fit to the data under these conditions and the probable pressure of helium gas was found to be $\sim 0.01 \mathrm{~atm}$. This was in line with our readings from the pressure gauge. At a pressure of $0.01 \mathrm{~atm}$, only protons with a kinetic energy below $\sim 80 \mathrm{KeV}$ could reach an equilibrium energy in the density of gas provided, as seen from our simulations in Fig. 14. 
For the $\mathrm{H}_{2}^{+}$beam energy of $1.6 \mathrm{MeV}$, no calibration runs were taken without the gas cell and accelerating grid structures. The window thickness was fixed in the $\mathrm{MC}$ at $350 \mathrm{~nm}$, and the proton beam energy was then extracted by fitting the time spectrum for a data run in which the gas was not flowing and the grid was turned off. The proton energy was found to be $760 \mathrm{KeV}$.

Two additional nominal $H_{2}^{+}$energies, 1.3 and $1.5 \mathrm{MeV}$ were used. However, the conditions were not varied for these energies and single low statistic runs were taken for each energy with the gas flowing and the accelerating field on. For each run, the proton beam energies were varied in the MC and then fit to the data distributions.

Cooled protons were searched for in TOF distributions of data runs with the gas on and the accelerating grid ramped up to produce a field of $60 \mathrm{KV} / \mathrm{m}$. The results are shown in Fig. [15] The narrow peak in all the distributions at $167 \mathrm{~ns}$ was the result of correlated noise in the DAQ system and was removed from the data. The long tails of background were expected to be flat or a slowly rising exponential with a large time constant. Cooled protons were expected populate a region of large TOF between 250 and $400 \mathrm{~ns}$. The background was fit using an exponential in the region of TOF greater than $500 \mathrm{~ns}$ and TOF less than 10 to $50 \mathrm{~ns}$, depending on the proton beam energy, where no protons were expected from the MC. The 1.3, 1.44 and $1.5 \mathrm{MeV} H_{2}^{+}$data background regions were consistent with being flat. The $1.6 \mathrm{MeV} \mathrm{H}_{2}^{+}$data set was the only data set with a background exponential fit with a positive time constant. The background (including correlated noise) subtracted data is shown in Fig. [16]

The MC curve was normalized by fitting the data in the time range of 0 to $400 \mathrm{~ns}$, which corresponds to the protons which do not achieve the equilibrium energy. The MC expectation was then calculated by integrating the number of events over a time window. The results are summarized in Table 2 We note that the MC expectations yield a very small number of cooled protons. This is in large part due to the effective thickness of the carbon windows. The data is consistent with no observation of cooled protons, but also compatible with the expectation from the simulations within the statistical errors.

\section{Experimental Challenges and Outlook}

The acceptance (including efficiency) can be estimated from the ratio of the MCP to the Si detector rates, and was about $0.01 \%$. The factors entering into this number include the geometrical acceptance for passage through the two $2.3 \mathrm{~mm}$ windows, possible misalignments of the windows with the MCP, and the MCP detector efficiency. Assuming perfect alignment, a $100 \%$ MCP detector efficiency, and a $55 \%$ transmission probability through the windows due to the Nickel grid, we calculate an acceptance using our detailed simulations of $0.4 \%$ for low energy protons. The remaining loss in acceptance was presumably from misalignment effects and MCP detector inefficiency. A magnetic field would have considerably increased the acceptance. For example, we calculate an acceptance increase of a factor 25 with a $5 \mathrm{~T}$ field. 
A further improvement in the experiment would be to remove the windows entirely. The effective window thickness measured in this experiment was $350 \mathrm{~nm}$, as obtained from a comparison of data and simulation results for proton energy spectra with and without windows present. The cause of the larger effective window thickness is not known, but may have been due to extensive exposure to the atmosphere. The effective column density seen by the protons from the exit window was similar to that from the helium gas. The exit window therefore set a threshold on the minimum energy which could be extracted from the gas cell and prevented us from observing low energy protons. Those protons which had enough energy to pass through the exit window were too energetic to be cooled in the gas cell.

We are preparing a new experiment in which a gas cell will be placed in a strong solenoidal field. A silicon drift detector will be placed inside the gas cell, and will directly measure the proton energies without the need for windows. The large increase in acceptance and sensitivity to low energies should therefore allow the observation of cooled protons.

\section{Conclusions}

This experiment was performed to study Frictional Cooling using protons. The number of cooled protons observed under various conditions was consistent with zero within large statistical errors. This result was explained by the small acceptance of the system and the large exit window thickness of our gas cell.

The data allowed for the tuning of untested simulation code. The experimental experience and the refined simulations will be used in the design and implementation of future experiments.

\section{Acknowledgements}

This work was funded through an NSF grant number NSF PHY01-04619, Subaward Number 39517-6653. Special thanks to S. Schlenstedt and H. Abramowicz for their contributions to our simulation efforts. We owe special gratitude to the RARAF staff and especially S. Marino, for their efforts in delivering the beam to the experiment.

\section{References}

[1] M.Muhlbauer et al., Nucl.Phys.Proc.Suppl. 51A, 135-142 (1996).

[2] Y.Nakai, T.Shirai, T.Tabata and R.Ito, At. Data Nucl. Data Tables 37, 69 (1987).

[3] W.H.Barkas, W.Birnbaum and F.M.Smith, Phys. Rev. 101, 778 (1956).

[4] J.S.Cohen, Phys. Rev. A 62, 022512 (2000). 
[5] E.Everhart, G.Stone and R.J.Carbone, Phys. Rev. 99, 1287 (1955).

[6] "The Stopping and Range of Ions in Matter", J.F.Ziegler, J.P.Biersack and U.Littmark, Pergamon Press (1985). Calculations were performed with SRIM-2003 version 19 (see http://www.SRIM.org).

[7] ICRU Report 49, Stopping Powers and Ranges for Protons and Alpha Particles, Issued 15 May, 1993 (see http://physics.nist.gov/PhysRefData/Star/Text). 


\section{Helium}

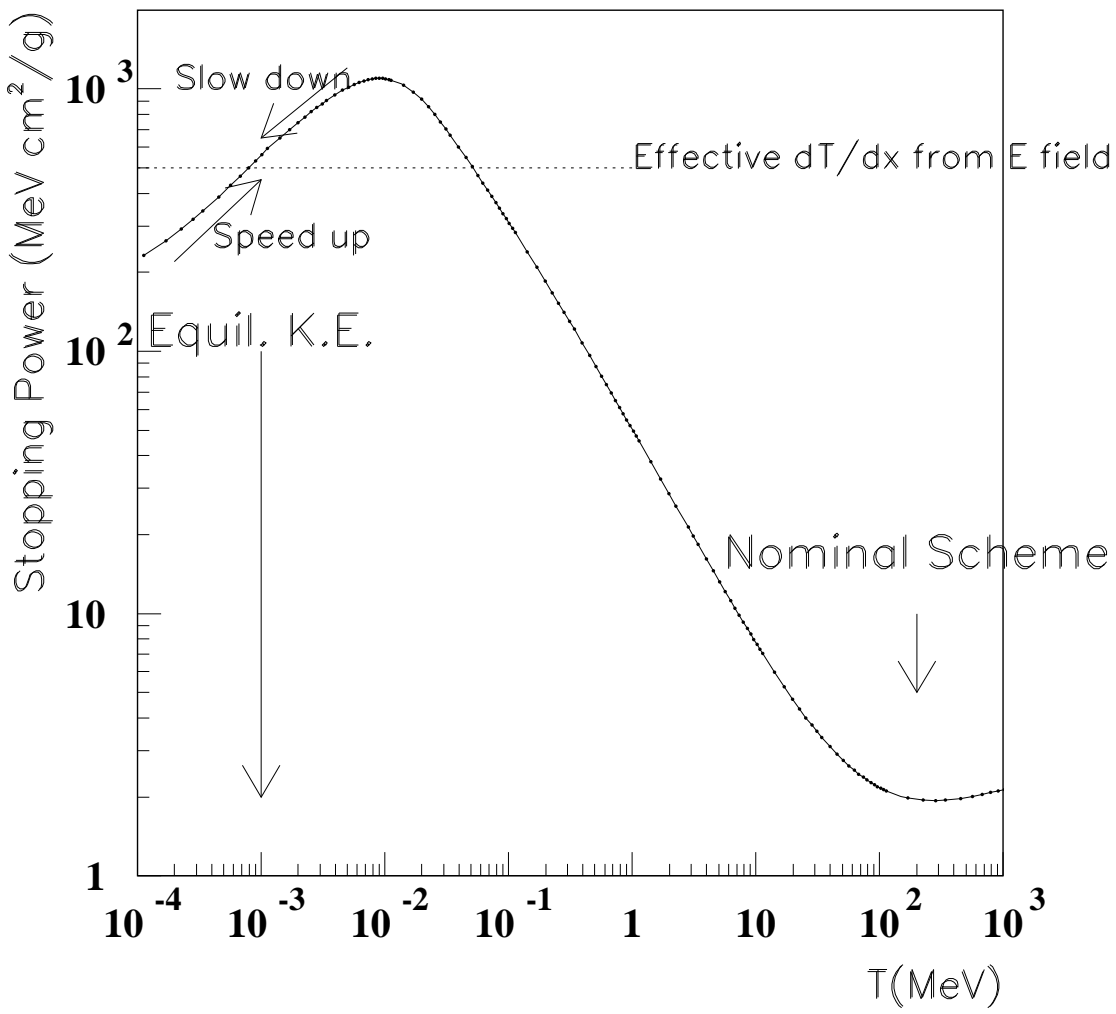

Figure 1: Stopping power $\left(\frac{1}{\rho} \mathrm{dT} / \mathrm{dx}\right)$ in helium as a function of kinetic energy, $\mathrm{T}$, for $\mu^{+}$(scaled from the NIST PSTAR tables[7]). The effective accelerating force resulting from an external electric field is superimposed. An equilibrium kinetic energy near $1 \mathrm{KeV}$ would result. The nominal scheme discussed for a Neutrino Factory would cool muons near $\mathrm{T}=200 \mathrm{MeV}$. 


\section{HELIUM}

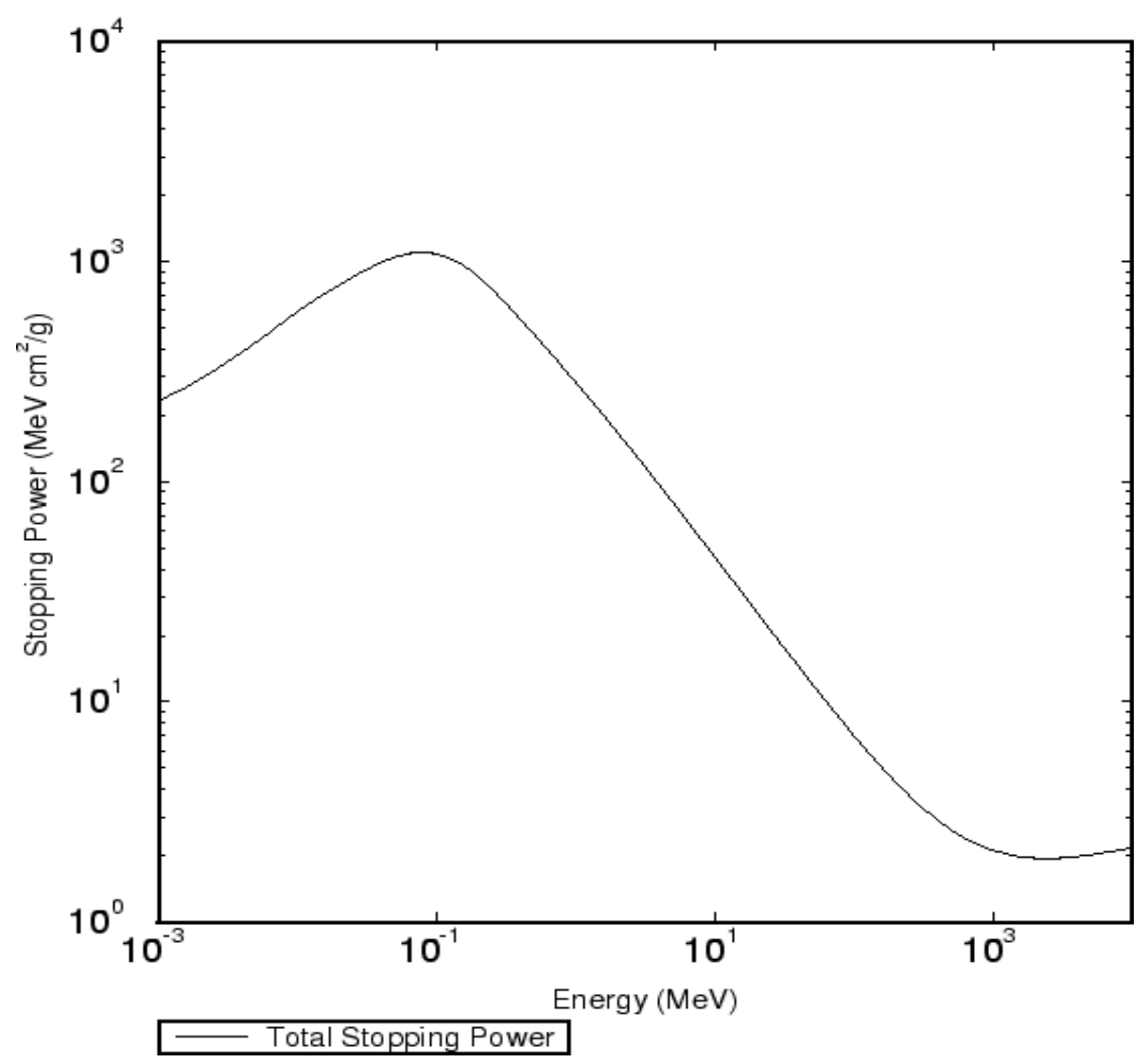

Figure 2: $\frac{1}{\rho} \mathrm{dT} / \mathrm{dx}$ in helium as a function of kinetic energy for protons in helium [7. 


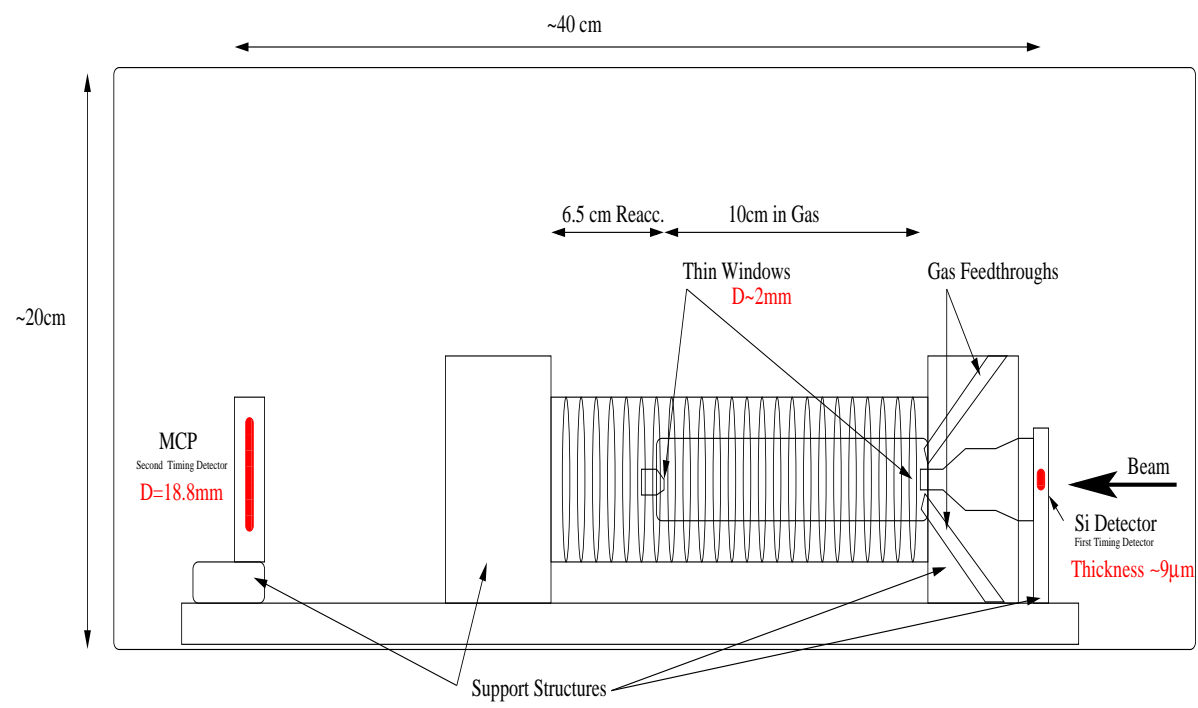

Figure 3: Schematic of the RARAF TOF experiment setup. The $H_{2}^{+}$beam comes in from the right and breaks up inside the first timing detector. The protons then pass through the gas cell which is surrounded by an accelerating grid. Those protons which survive through the exit window of the gas cell are reaccelerated by an accelerating grid, which extends beyond the gas cell, and drift toward the second timing detector.
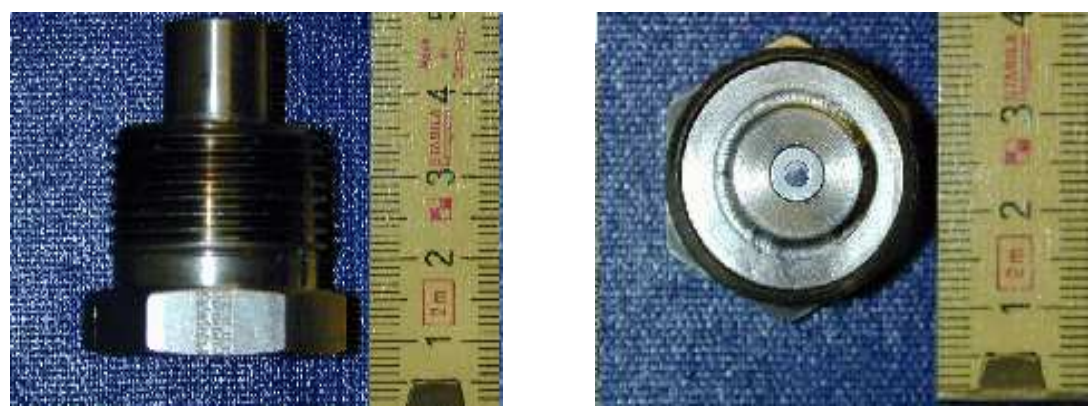

Figure 4: Photographs of the entrance window holder. The window is visible at the center of the right-hand picture. 


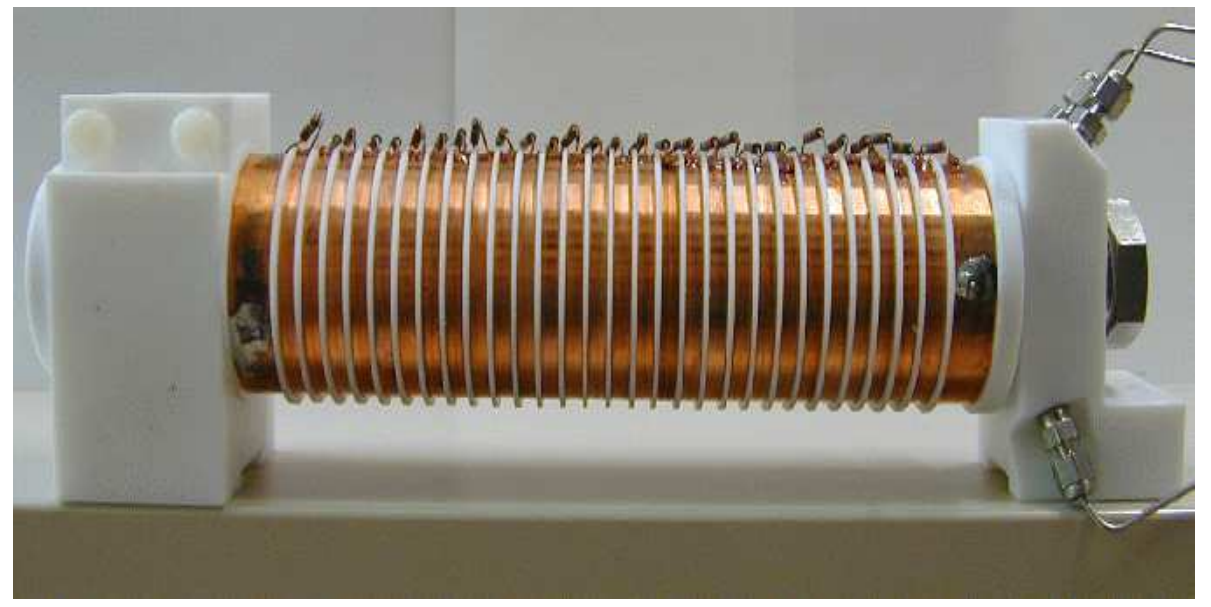

Figure 5: Photograph of the gas cell surrounded by the accelerating grid. The accelerating grid is supported by Teflon blocks.

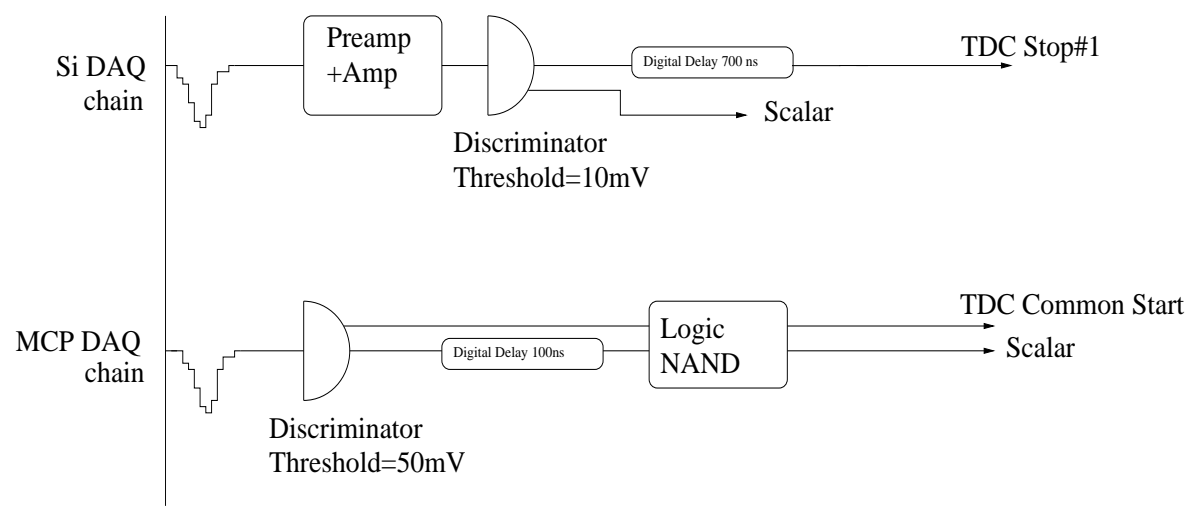

Figure 6: Schematic of the DAQ chain for the TOF measurement. 


\begin{tabular}{||l||l|l|l|l|l|l|l|l|l|l|l|l|l||}
\hline \hline $\begin{array}{l}\mathrm{T}\left(\mathrm{H}_{2}^{+}\right) \\
{[\mathrm{MeV}]}\end{array}$ & 1.44 & 1.44 & 1.44 & 1.44 & 1.44 & 1.44 & 1.44 & 1.6 & 1.6 & 1.6 & 1.6 & 1.5 & 1.3 \\
\hline He gas & No* $^{*}$ & No* $^{*}$ & No$^{*}$ & Yes & Yes & No & No & Yes & Yes & No & No & Yes & Yes \\
\hline $\begin{array}{l}\text { Acc. Grid } \\
(\sim 60 \mathrm{KV} / \mathrm{m})\end{array}$ & Off* & Off* & Off* & On & Off & On & Off & On & Off & On & Off & On & On \\
\hline $\begin{array}{l}\text { TOF Dist. } \\
{[\mathrm{cm}]}\end{array}$ & 38.5 & 48.5 & 68.5 & 38.5 & 38.5 & 38.5 & 38.5 & 38.5 & 38.5 & 38.5 & 38.5 & 38.5 & 38.5 \\
\hline$\#$ Events & $60 \mathrm{~K}$ & $31 \mathrm{~K}$ & $59 \mathrm{~K}$ & $31 \mathrm{~K}$ & $7 \mathrm{~K}$ & $4 \mathrm{~K}$ & $3 \mathrm{~K}$ & $81 \mathrm{~K}$ & $2 \mathrm{~K}$ & $3 \mathrm{~K}$ & $3 \mathrm{~K}$ & $1 \mathrm{~K}$ & $3 \mathrm{~K}$ \\
\hline \hline
\end{tabular}

Table 1: Summary of datasets taken. ${ }^{*}$ For these runs the entire gas cell and accelerating grid structures were removed from the beam line. 


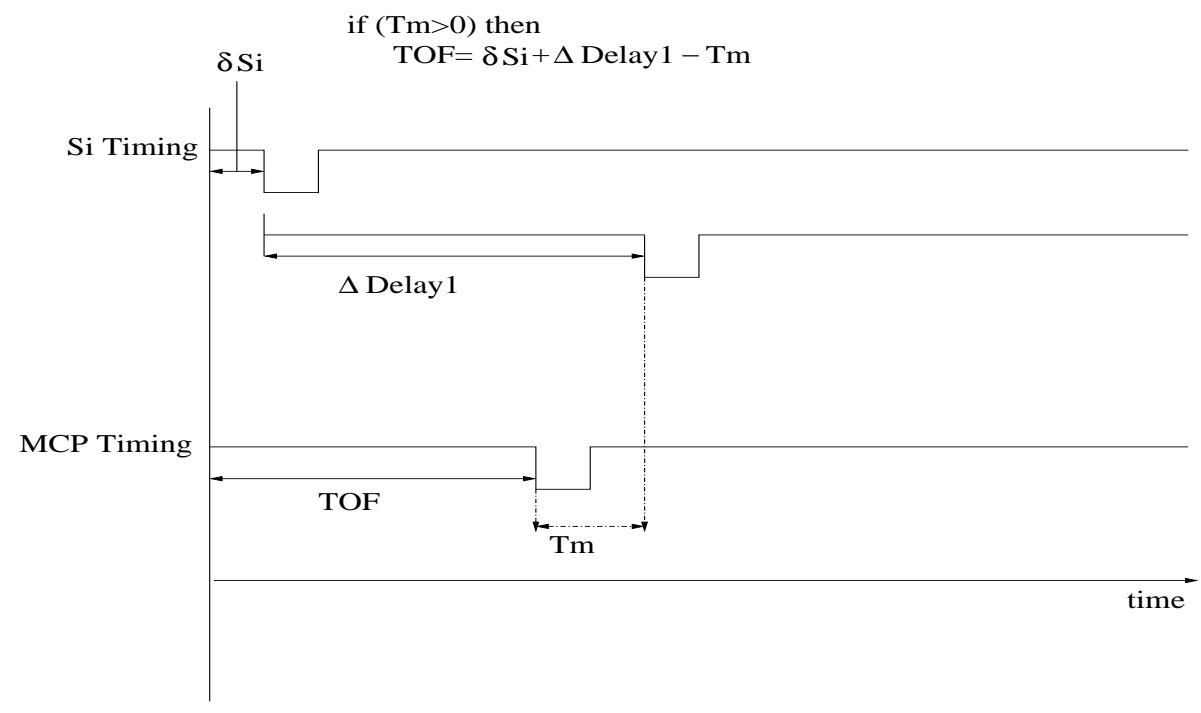

Figure 7: Trigger timing scheme.

\begin{tabular}{||c|c|c|c|}
\hline \hline $\begin{array}{c}\mathrm{H}_{2}^{+} \text {Beam } \\
\text { Energy (MeV) }\end{array}$ & $\begin{array}{c}\text { Fitted Proton Beam } \\
\text { Energy (KeV) }\end{array}$ & $\begin{array}{c}\sum_{250 \mathrm{~ns}}^{400 \mathrm{~ns} \text { Events }} \\
\text { (MC exp.) }\end{array}$ & $\begin{array}{c}\sum_{250 \mathrm{~ns}}^{750 \mathrm{~ns}} \text { Events } \\
\text { (MC exp.) }\end{array}$ \\
\hline \hline 1.3 & 710 & $-4 \pm 17(2)$ & $15 \pm 31(3)$ \\
\hline 1.44 & 721 & $-2 \pm 45(28)$ & $64 \pm 82(29)$ \\
\hline 1.5 & 745 & $-1 \pm 12(0)$ & $31 \pm 22(0)$ \\
\hline 1.6 & 760 & $63 \pm 94(0)$ & $185 \pm 176(0)$ \\
\hline \hline
\end{tabular}

Table 2: Results of data runs. Note that the MC expectation does not include the possible variation of the MCP efficiency with energy. MC expectation is given assuming $0.01 \mathrm{~atm}$ of helium gas and $350 \mathrm{~nm}$ thick carbon entrance and exit gas cell windows. 

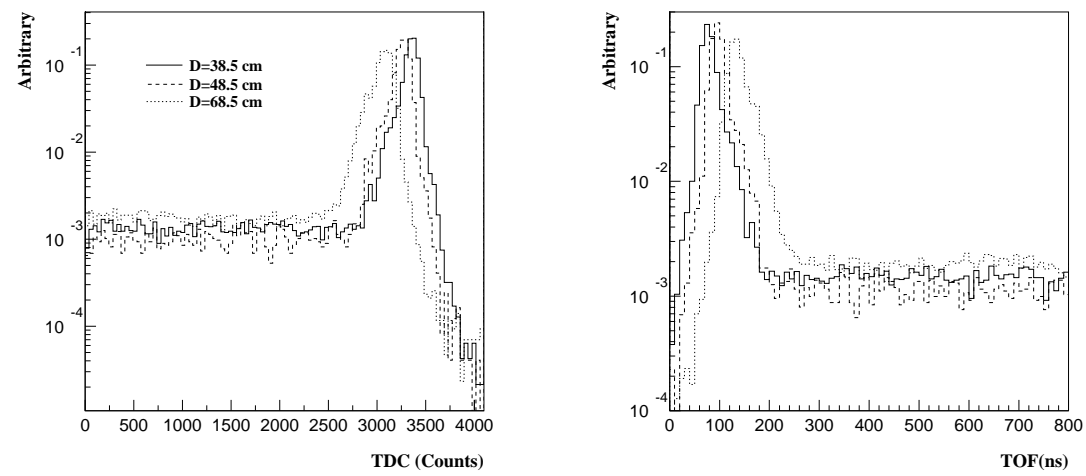

Figure 8: Data from varying the flight distance between detectors. The histograms are normalized to area for comparison. (left) Raw TDC counts. (right) Reconstructed time in (ns). 


\section{T0 Calibration}

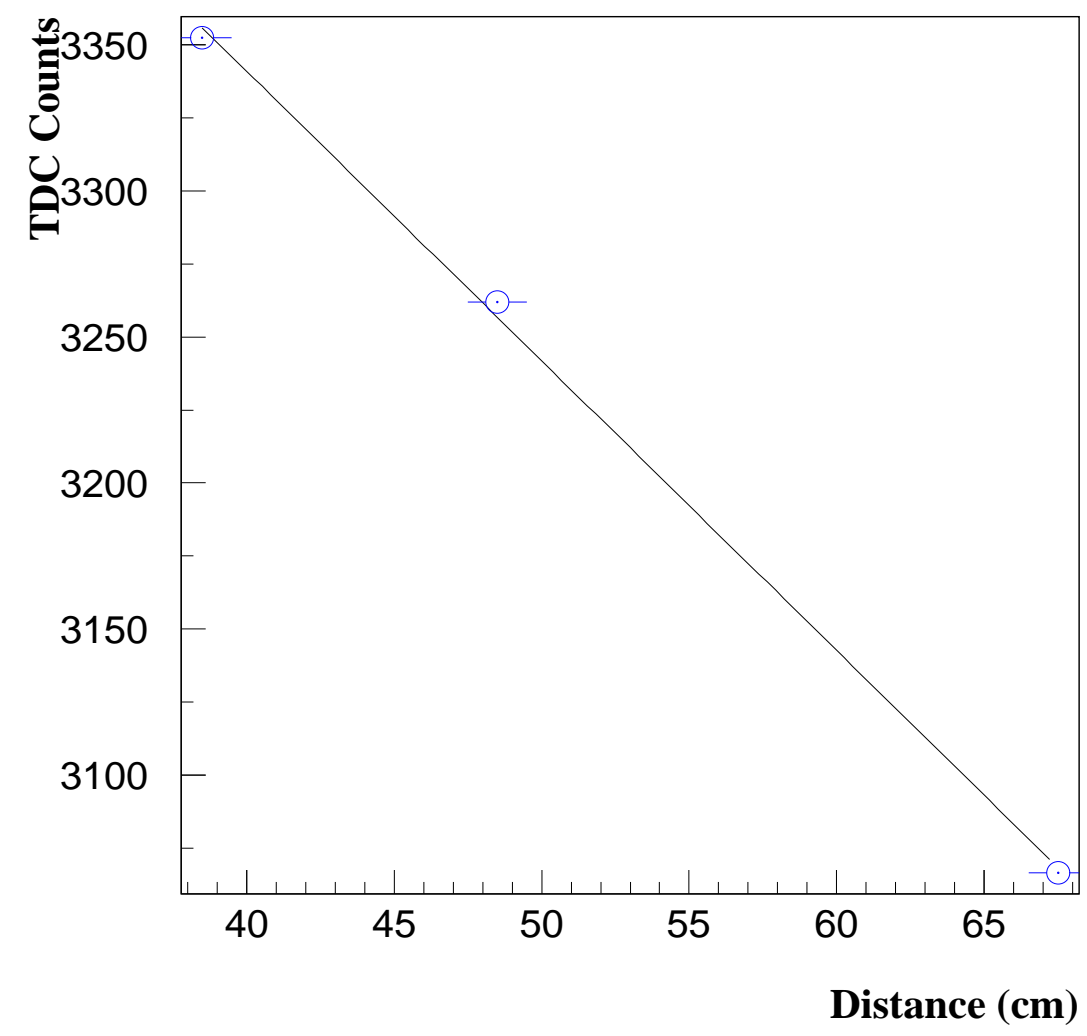

Figure 9: Determination of time offset in TDC Counts for the DAQ chain. 


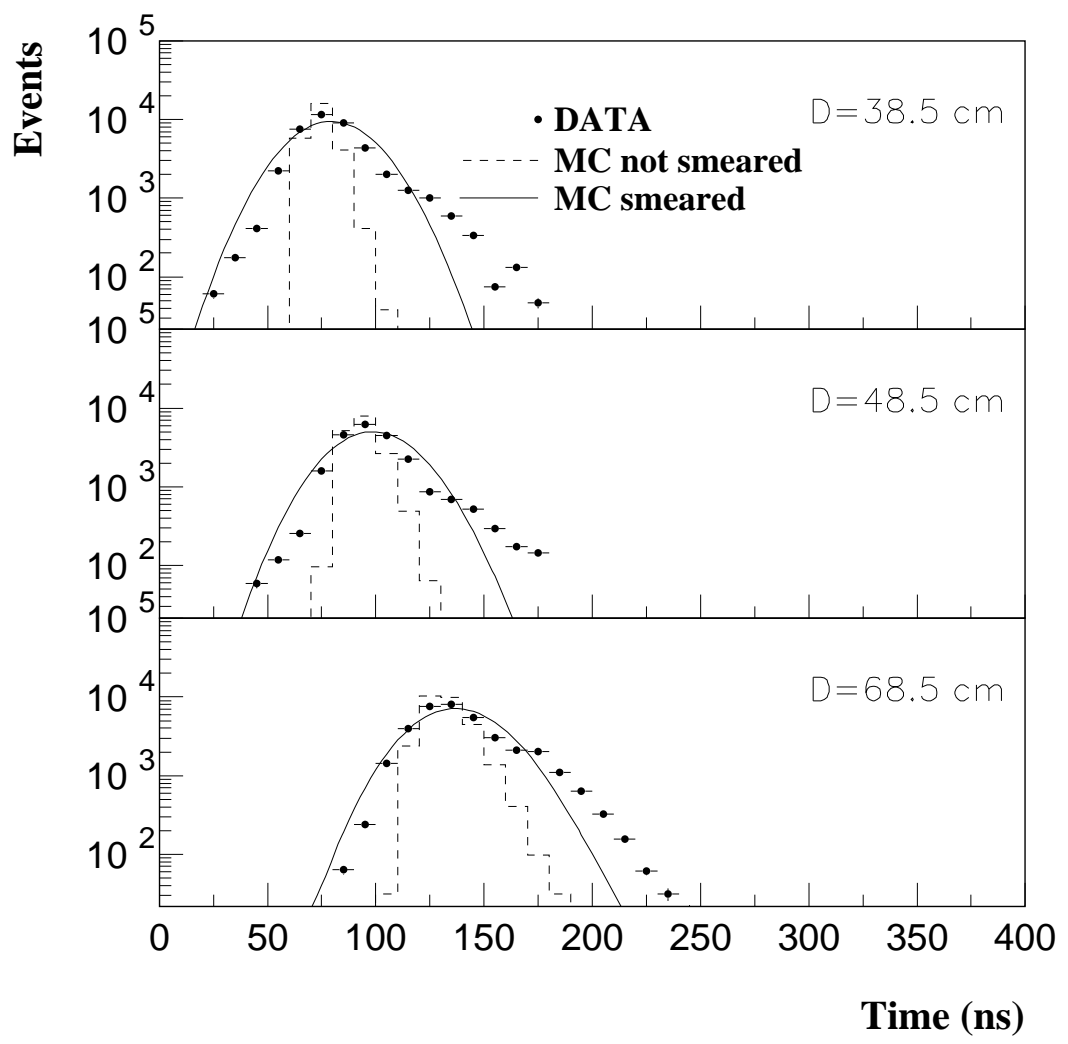

Figure 10: Reconstructed time distributions for three flight distances. The dashed histogram is the MC expectation and the points are the data. The solid curve is a fit to the data by a convolution of the $\mathrm{MC}$ expectation with a gaussian, whose width represents the timing resolution of the system. 


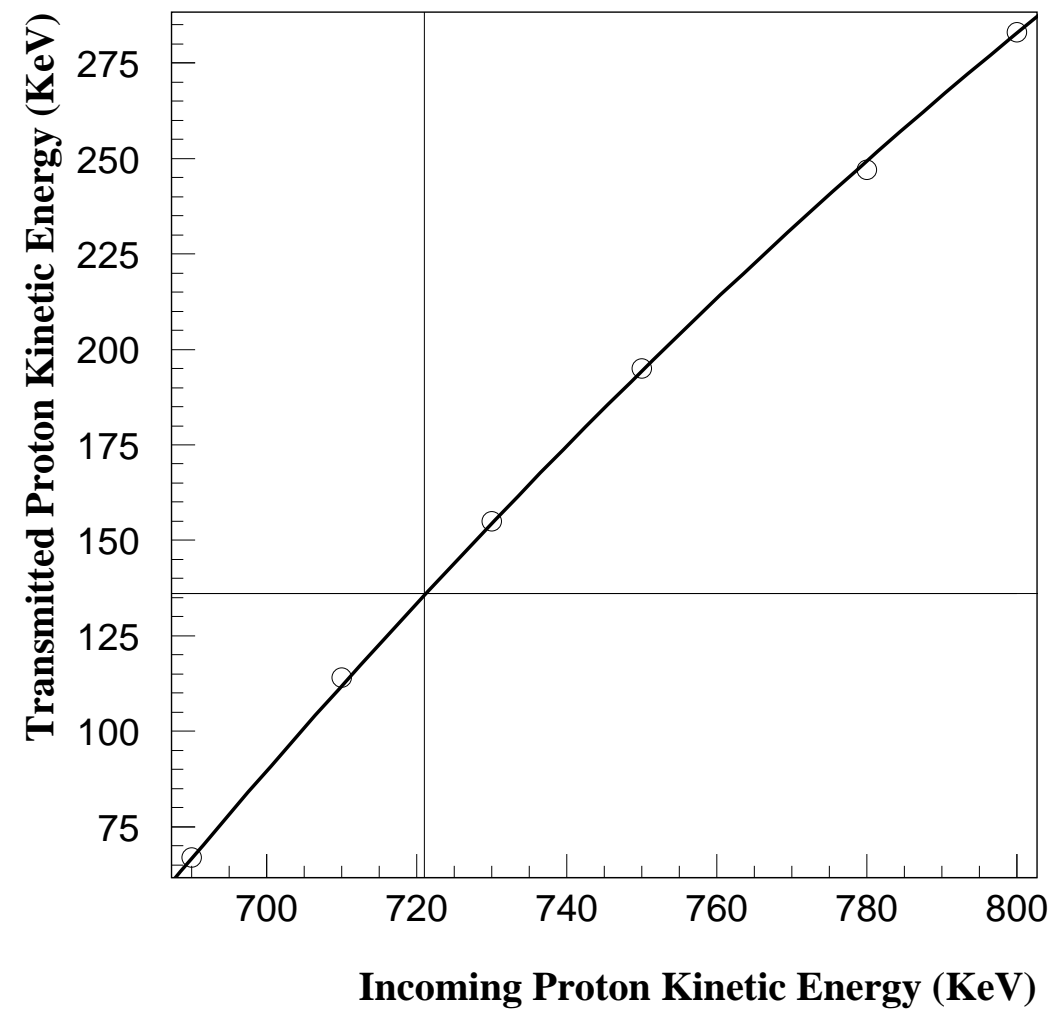

Figure 11: The proton transmitted kinetic energy spectrum through $9 \mu \mathrm{m}$ of silicon, as a function of the incoming proton kinetic energy from SRIM calculations. 


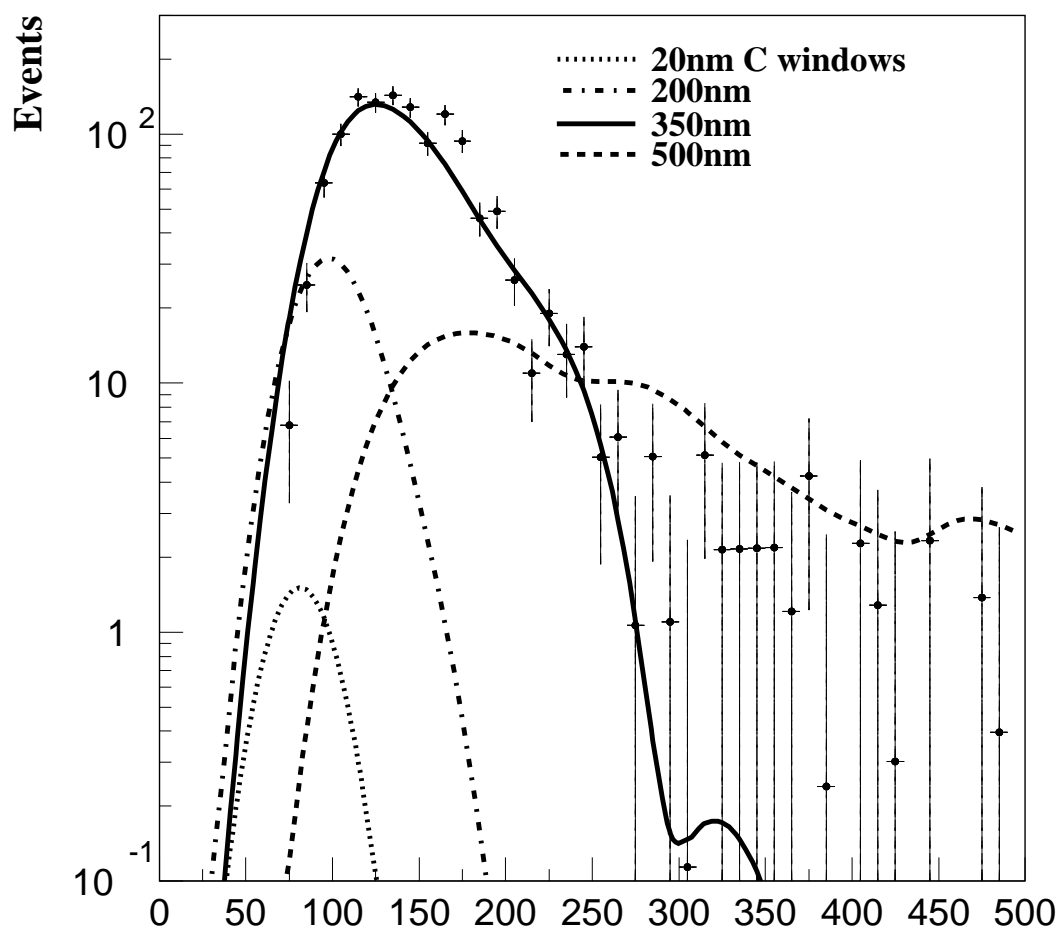

Time(ns)

Figure 12: Measured time spectrum for a data run consisting of a beam of $1.44 \mathrm{MeV} \mathrm{H} H_{2}^{+}$(721 KeV protons) with no gas flowing in the gas cell and no accelerating potential. The curves correspond to MC spectra with different gas window thicknesses. The normalization of the curves was performed via a fit to the data in the time window between 0 and $400 \mathrm{~ns}$. 


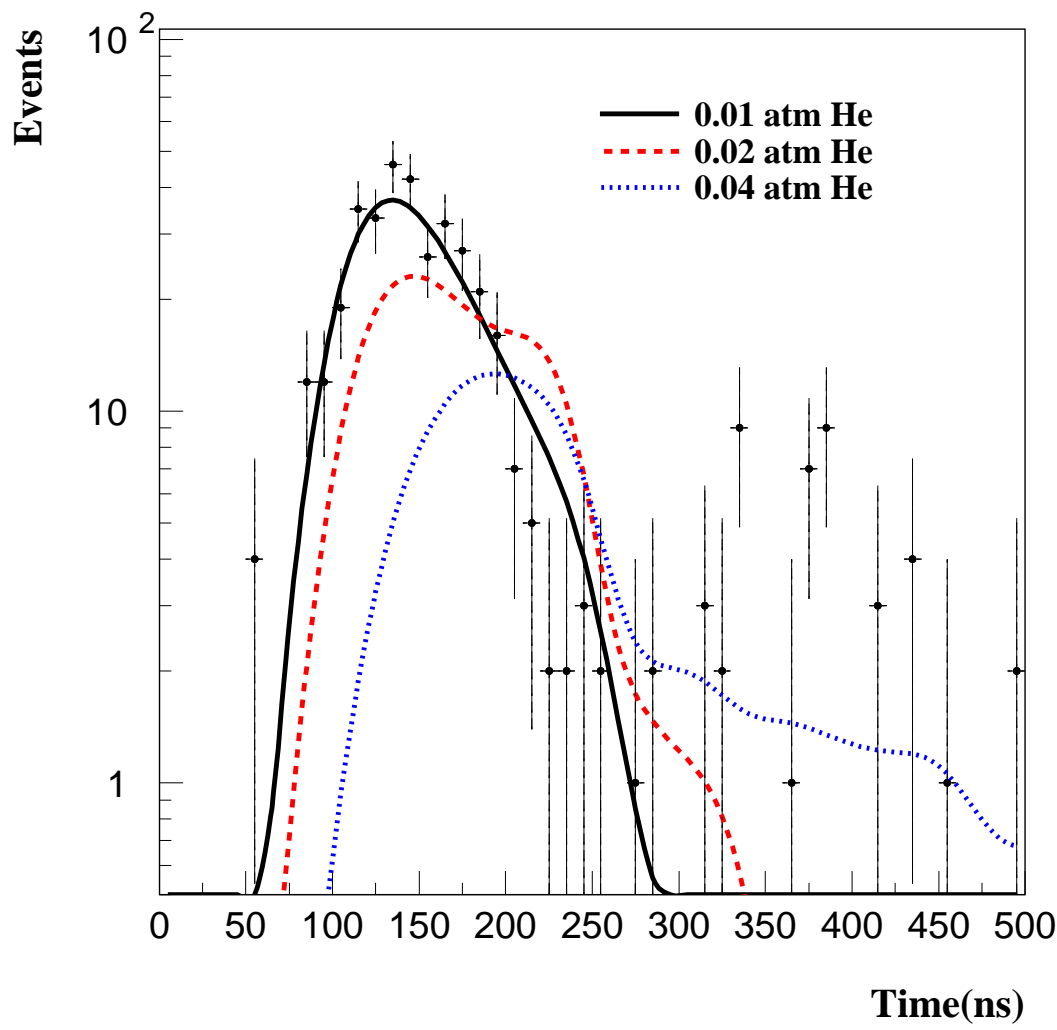

Figure 13: Measured time spectrum for a data run consisting of a beam of $1.44 \mathrm{MeV} H_{2}^{+}$(721 KeV protons) with gas flowing and no accelerating potential. The curves correspond to MC spectra with varying pressures and $350 \mathrm{~nm}$ thick carbon gas cell windows. The normalization of the curves was performed by fitting to the data in the time window between 0 and $400 \mathrm{~ns}$. 


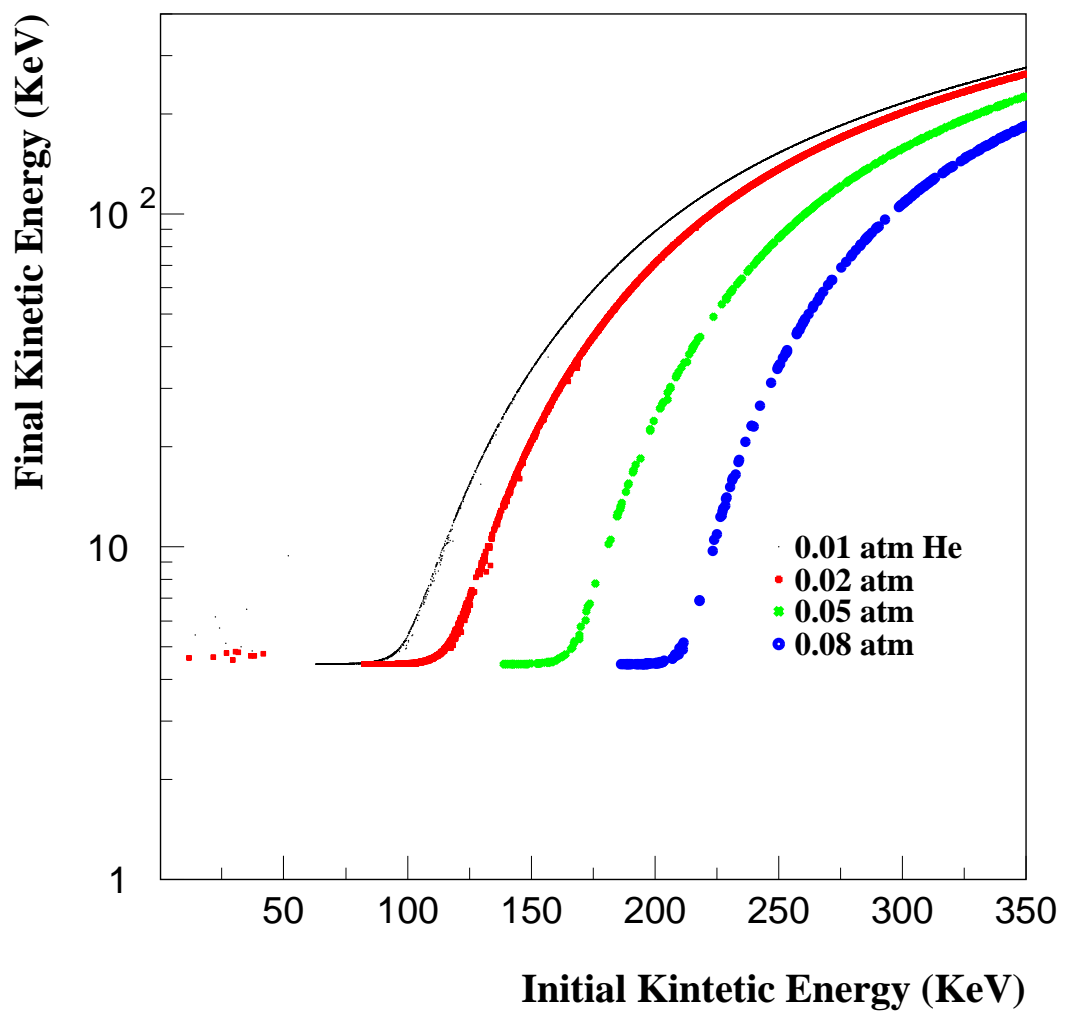

Figure 14: $\mathrm{MC}$ simulations of proton final kinetic energies as a function of initial kinetic energies for an accelerating potential of $60 \mathrm{KV} / \mathrm{m}$ at various gas pressures. The minimum final kinetic energy is fixed by the potential drop after the gas cell. 

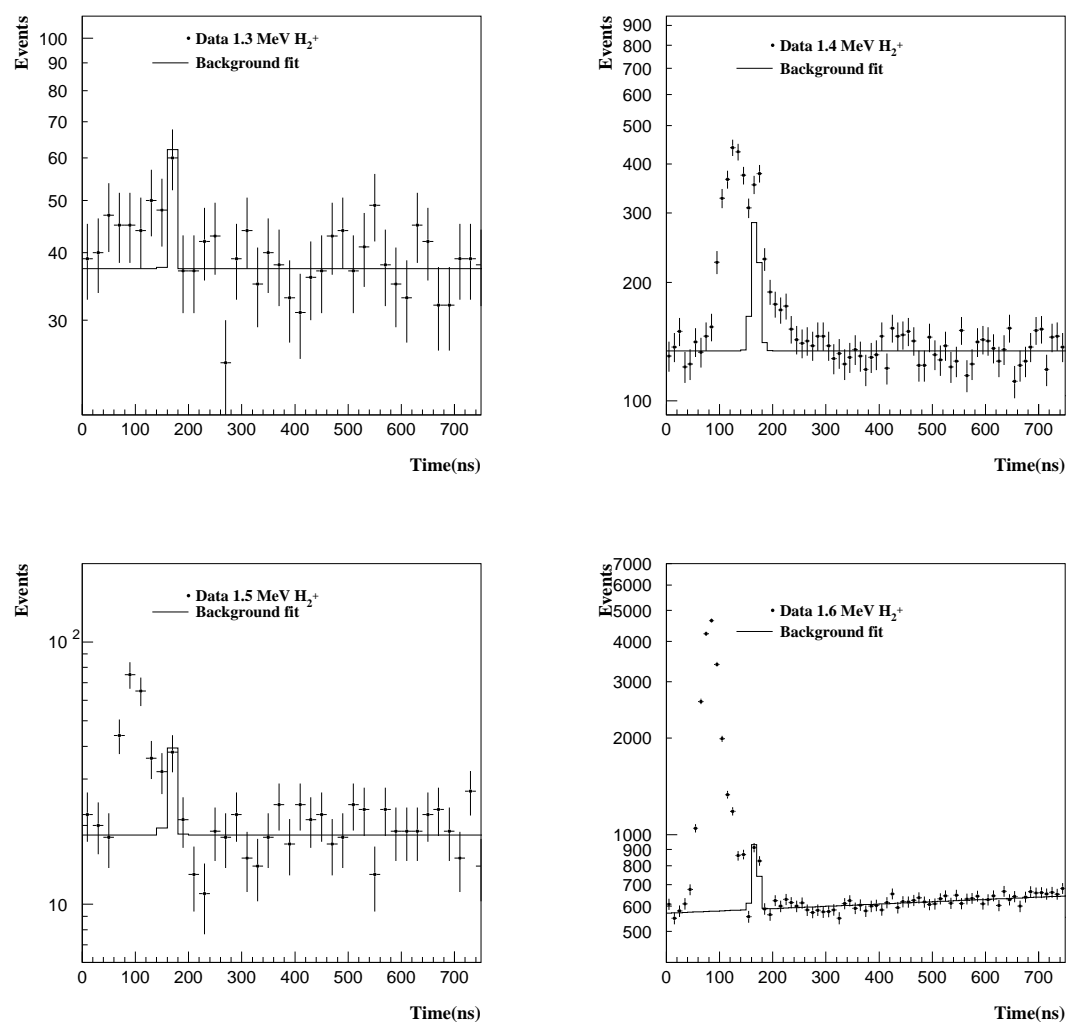

Figure 15: Measured time distributions for data runs with an accelerating potential of $60 \mathrm{KV} / \mathrm{m}$ and gas flowing. The bump at time $=167 \mathrm{~ns}$ is from correlated noise. The 1.3 and $1.5 \mathrm{MeV} \mathrm{H}_{2}^{+}$data sets are plotted with coarser bin size as a result of their low statistics. 

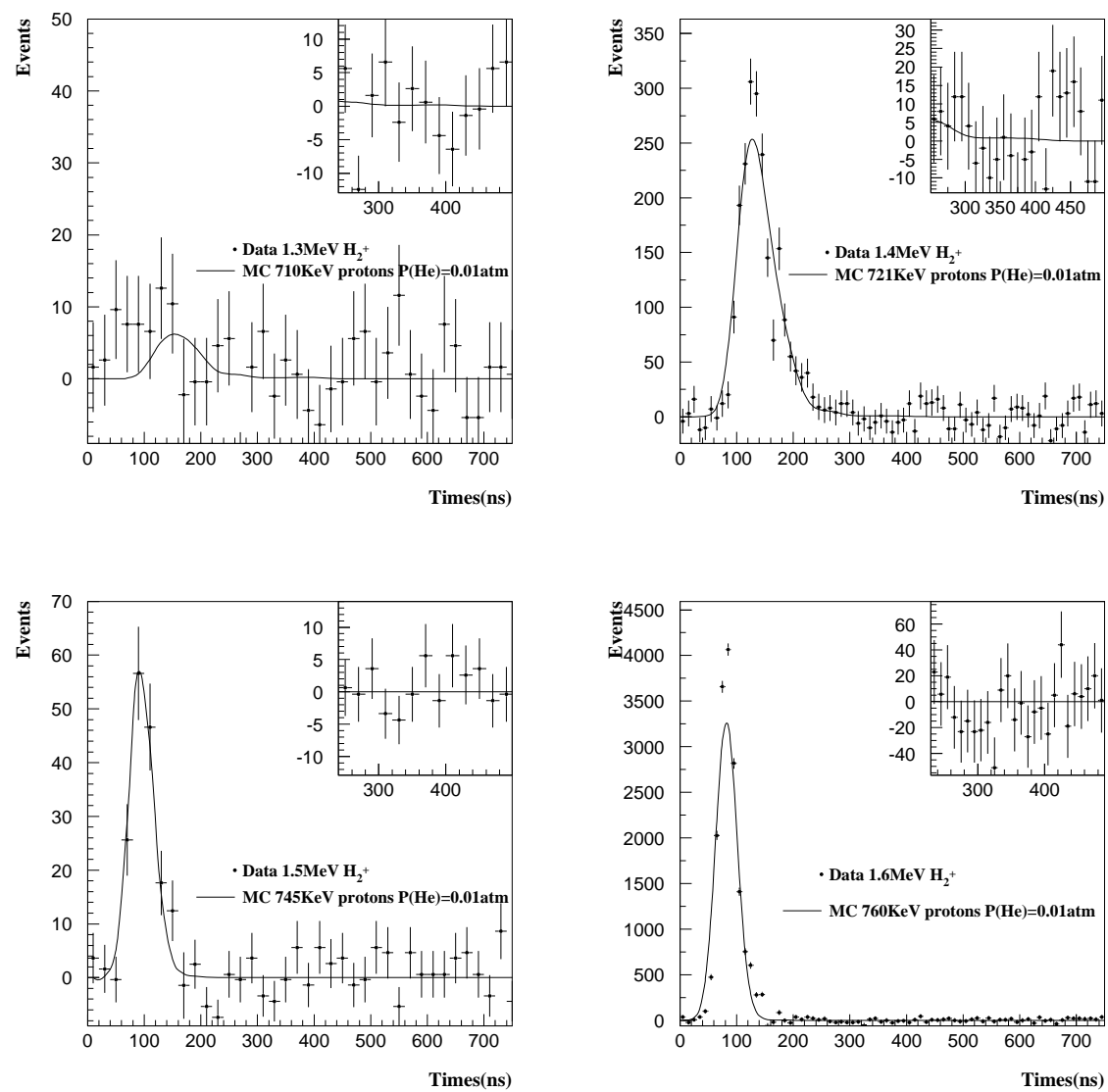

Figure 16: Measured time distributions after subtraction of the background. The solid curves are predictions from our MC simulations with $350 \mathrm{~nm}$ thick carbon entrance and exit windows. The 1.3 and $1.5 \mathrm{MeV} H_{2}^{+}$data sets are plotted with coarser bin size as a result of their low statistics. 